\title{
JOGO DE EMPRESAS EM GESTÃO DE PROJETOS: APLICAÇÃO EM UMA MULTINACIONAL AUTOMOBILÍSTICA
}

\section{Eduardo de Lima Pinto Carreiro}

DOI: http://dx.doi.org/10.12712/rpca.v9i4.593

eduardolpc@id.uff.br

Universidade Federal Fluminense (UFF), Volta Redonda /RJ, BRASIL

\section{Murilo Alvarenga Oliveira}

malvarenga@id.uff.br

Universidade Federal Fluminense (UFF), Volta Redonda /RJ, BRASIL

\section{Resumo}

O estudo teve como objetivo analisar a opinião de profissionais quanto às potencialidades de um jogo de empresas dinamizado por um simulador organizacional para a gestão de projetos na formação de competências. A pesquisa de cunho descritivo foi realizada por meio de um levantamento de opinião com 21 profissionais da área de projetos de uma multinacional do setor automobilístico. O instrumento de coleta de dados foi baseado nos estudos de Carvalho e Rabechini Jr (2006) que analisaram as habilidades relevantes em gestão de projetos sob três aspectos: o gestor, a equipe e o contexto da organização para o desenvolvimento de projetos. No que se refere a abordagem do tratamento dos dados, o estudo utilizou-se de uma abordagem quantitativa. As opiniões dos profissionais revelaram a existência de distintas impressões quanto às contribuições da atividade vivencial na formação de competências. Foi possível perceber que as características dos grupos de profissionais de acordo com o perfil associado a experiência e cargo, determina uma avaliação diferente. De forma geral, os participantes indicaram que a atividade dinamizada pelo jogo de empresas promove contribuições na formação de competências em projetos.

Palavras-chave: Gestão de projetos. Jogos de empresas. Simuladores organizacionais.

\section{Abstract}

The study analyzed the opinion of professionals about the potential of a business game spurred by an organizational simulator for project management skills training. The descriptive research was conducted through a survey with 21 professionals projects of a multinational automotive industry. The relevant skills were analyzed in project management from three aspects: the manager, the staff and the organization's context for development projects. As regards the data processing approach, the study used a quantitative analysis in the responses measures. The opinions of the professionals have revealed distinct impression of the contributions of experiential activity in skills training. It could be observed that the characteristics of professional groups according to the profile associated with experience and position, determines a different assessment. In general, the participants indicated that the streamlined activity by business game help in development in skills training projects.

Keywords: Project management. Business game. Management Simulators. 


\section{Introdução}

Este estudo descreve a aplicação de um treinamento para formação de gerentes de projetos, dinamizada por um jogo de empresas, cujo simulador organizacional foi desenvolvido especificamente para a realidade de uma multinacional automobilística instalada no Brasil. Neste sentido, o propósito da pesquisa foi analisar a contribuição de uma atividade vivencial mediada por um jogo de empresas funcional na formação de competências em gestão de projetos, segundo a opinião dos participantes.

Como gerenciar projetos pode ser entendido em realizar algo complexo (no tempo, dentro do orçamento e com a especificação) por meio das pessoas, as quais representam uma prerrogativa fundamental da gestão em geral (HOLT, 1987). Neste sentido as organizações dependem cada vez mais dos projetos para a realização de suas atividades comerciais (BARTSCH, EBERS; MAURE, 2013), sendo reconhecido como um mecanismo fundamental para iniciativas de negócios e operações (MARTIN, 2000).

Desde sua fundação em 1969, nos EUA, o Project Management Institute (PMI) tem como propósito a prática e o desenvolvimento dos conjuntos de conhecimentos que envolvem a profissão de gerenciamento de projetos. Mediante seu Guia do Project Management Body of Knowledge (PMBOK), o qual se tornou a principal referência em gestão de projetos, sendo a certificação de Profissional em Gerenciamento de Projetos (PMP) muito requisitada por especialistas da área, pois tal credenciamento é reconhecido em todo o mundo porquanto significa experiência, realização educacional e conhecimento profissional, requisitos essenciais para a prática competente de um gerente de projetos (PMI, 2013a).

Para ilustrar a importância da área no final de 1970 havia cerca de dois mil profissionais credenciados, no início do século XXI contava com quarenta mil profissionais. Dessa forma, o PMI já representava, em 2011, a maior associação do gênero no mundo, com mais de 260.000 membros em cerca de 170 países e a partir do ano de 2013 conta com cerca de setecentos mil membros credenciados (PMI, 2013b).

A preocupação do PMI em promover a formações dos gestores de projetos, em adquirir conhecimento e em entregar resultados é explícita em todas as suas ações, desde cursos convencionais nos centros associados em todo o mundo até iniciativas envolvendo tecnologias digitais de educação para ampliar as oportunidades de aprendizagem. Dentre elas destacam-se apresentações, projetos em grupo, exercícios de simulação e de dinâmica, testes e exercícios colaborativos (PMI, 2013).

O próprio PMI (2013a), no capítulo que trata do gerenciamento de recursos, destaca a importância do gestor de projetos ao apresentar uma visão integrada, combinando habilidades técnicas e interpessoais onde os treinamentos indicados exploram as atividades colaborativas para a solução de problemas. Os aspectos abordados para a formação de gerentes de projetos são a comunicação, o gerenciamento de conflitos, a negociação e a liderança. Para tanto, há uma sugestão de treinamentos com equipes virtuais, cujo objetivo é compartilhar a execução de papéis à distância, não obstante, reforçando a resolução de problemas coletivamente.

Como a formação do gerente de projetos demanda uma integração entre teoria e prática, tal e qual as indicações do PMI (2013a) para a gestão de equipes de projetos baseada na experiência nas organizações, pode-se associar tais demandas com a utilização de jogos de empresas. Em seu estudo Oliveira (2009a) destaca a importância dos jogos de empresas para a formação gerencial, indicando os principais benefícios desta técnica de aprendizagem, em que os tipos com maior incidência na literatura são: aprendizagem pelo praticante, envolvimento e motivação na atividade, tomada de decisão, visão sistêmica, integração teoriaprática e desenvolvimento de habilidade de liderança e de trabalho em equipe.

Martin (2000) identifica os problemas que surgem quando esse treinamento é realizado somente por meio de métodos tradicionais. Freeman e Dumas (1989) identificaram as lacunas entre a aprendizagem de livros didáticos e as circunstâncias operacionais que possuem na vida real. Larreche (1987) vê lacunas entre o conhecimento não traduzido e experimentação desinformada. Kotter (1982) argumenta que há descompasso entre a sabedoria convencional sobre as funções de gestão, das ferramentas e do sistemas e o comportamento gerencial real. November (1993) destaca a diferença entre as atividades realizadas pelo lado esquerdo e direito do cérebro, e identifica um desequilíbrio nos métodos de ensino da escola de negócios tradicionais. Esses autores defendem 
a contribuição potencial dos jogos e das simulações para abordar essas lacunas. $\mathrm{O}$ uso de jogos e de simulações é, segundo Martin (2000), consistente com o pensamento por trás de métodos de ensino inovadores. Certos tipos de conhecimentos não podem ser facilmente aprendidos lendo, escrevendo ou refletindo. Faz-se necessário o aprendizado vivencial, sendo alguns métodos de ensino e de aprendizagem projetados para fornecerem subsídios para a experiência direta, por exemplo: estudos de caso, jogos, simulações e dramatizações (MARTN, 2000). Peters e Homer (1996) afirmam que as pessoas aprendem habilidades de gerenciamento de projetos sob pressão, fazendo, pela luta, avaliando boas e más ações, ou seja, é a ferramenta para aumentar a eficiência do meio de aprendizagem. Wateridge (1997) reconhece a contribuição fundamental da aprendizagem experiencial de formação em gestão de projetos.

Realizou-se a pesquisa em uma empresa multinacional no ramo automobilístico, com 21 responsáveis pela a área de gestão de projetos e analisou-se a opinião desses profissionais quanto às potencialidades de uma atividade vivencial que utilizou um jogo de empresas dinamizado por um simulador organizacional para a gestão de projetos, denominado PMS-SIM (Project Management Simulation), para a contribuição na formação de competências em gestão de projetos.

Diante das necessidades de uma formação integrada indicadas pelo PMI, inclusive a adoção de técnicas de cunho prático para assimilação dos conhecimentos estipulado no PMBOK e do próprio contexto da pesquisa, o problema identificado para guiar o estudo pode ser estabelecido na seguinte questão: Qual a impressão dos gestores de projetos sobre o uso de ACP (Abordagem Centrada no Participante) na formação de competência na área? Para responder a esta questão, o estudo teve como objetivo: analisar a existência de diferenças nas impressões conforme o perfil (nível hierárquico) do profissional.

\section{Fundamentação Teórica}

\section{Formação de competência na gestão de projetos}

A disciplina de gerenciamento de projetos pode ser vista como um elemento importante de gestão, da mesma maneira que a educação em diversas áreas com destaque na Administração e na Engenharia. A gestão de projetos está sendo reconhecido como um mecanismo fundamental para iniciativas de negócios e de operações cada vez mais instáveis e sob condições de incerteza (GILBREATH, 1988). Existem as iniciativas para a formação de aprendizagem em gerentes de projeto experientes e potenciais a partir de aspectos quantitativos e, habilidades qualitativas, além das questões sociais envolvidas na gestão de projetos.

Nos últimos anos, o tema competência, seu desenvolvimento e sua gestão, está evidente nas listas de discussões acadêmicas e empresariais, aliado a diferentes instâncias de percepção: no nível da pessoa das organizações e dos países (FLEURY e FLEURY; 2004).

Para McClelland (1973) competência pode ser definida como uma característica implícita a pessoa que é eventualmente associada a um melhor desempenho na execução de uma atividade ou em alguma situação.

Frame (1999) sugere que competência em gestão de projetos não se limita exclusivamente ao âmbito do indivíduo, dividindo-se em três tipos: as individuais, as de equipe e as da organização. O primeiro tipo, segundo Fleury e Fleury (2004), seria o saber agir responsável e reconhecido, que implica mobilizar, integrar, transpassar conhecimentos, recursos e habilidades, que adicionem valor econômico à organização. As competências de equipe, segundo tipo, Frame (1999) define como a capacidade de solucionar problemas complexos em âmbito multidisciplinar. Já as competências da organização formam o terceiro tipo, que Frame (1999) afirma ser capacidade de construção de um ambiente que permita a participação dos indivíduos e das equipes, possibilitando o desenvolvimento de projetos de forma eficaz.

Carvalho e Rabechini Jr (2006) afirmam que as competências individuais podem ser evidenciadas com mais detalhes pelo modelo de stakeholders de Clealand (1999) que o dividiu em dois grupos: primários e secundários. Os primários são formados pelo gerente de projetos, pelo patrocinador, pelos técnicos, pelo gerente funcional e de suporte. Os secundários podem relacionar o governo e as subcontratadas.

Rabechini Jr e Carvalho (1999) estudaram as habilidades relevantes em gestão de projetos sob três aspectos: o gestor, a equipe e o contexto da organização para o desenvolvimento de projetos, verificando, em seus estudos, que algumas habilidades das equipes confluíam 
em pontos relacionados a liderança, a facilidade de negociação e a capacidade técnica.

Martin (2000) lista em seu trabalho as principais habilidades do gerente de projetos: planejamento, organização, comando, coordenação, controle, tomada de decisão, negociação, habilidades interpessoais, gestão do tempo, gestão de recursos e ferramentas de projetos, ou seja, gestão de projetos é fazer algo complexo acontecer (no tempo, dentro do orçamento e como a especificação).

Fleury e Fleury (2004) confirmam o conceito de Martin (2000), mas subdividem as competências individuais em três tipos: competências de negócio, competências técnicas e competências sociais. As competências de negócio são compostas da visão estratégica do empreendimento e da capacidade de planejamento. Competências técnicas seriam os conhecimentos específicos. Por último, as competências sociais que são habilidades de comunicação, negociação e trabalho em equipe.

\section{Jogos de empresas na aprendizagem gerencial}

Para Sauaia (2008), os jogos de empresas são baseados em um modelo de simulação no qual as características das empresas e do ambiente em que ela está inserida são retratadas de maneira aproximada das situações reais, de modo que as decisões empresarias possam ser colocadas em prática. Desta maneira, por meio dos jogos de empresas, pode-se observar como os indivíduos processam as informações recebidas e tomam decisões num ambiente similar ao de uma organização. Em se tratando da formação em gestão, uma das técnicas adotadas para desenvolvimento de habilidades de tomada de decisão são os jogos de empresas (MOTTA, QUINTELLA; ARMONDDE-MELO, 2012; SAUAIA e OLIVEIRA, 2011; OLIVEIRA, 2009). Os jogos de empresas são um exercício de tomada de decisões, reproduzindo de forma simplificada e parcial uma situação real (SAUAIA, 2009).

Um artigo clássico que apresenta o campo de pesquisa em jogos de empresas foi desenvolvido por Keys e Wolfe (1990). Nele os autores apresentam uma ampla descrição de mais de quarenta anos de pesquisas desenvolvidas em diversas áreas como: eficiência educacional da técnica, ambientes de aprendizagem, análise de simuladores, criação de contexto para realização de pesquisas, teste de modelos gerenciais e o surgimento de laboratórios de gestão (SAUAIA, 2008; JENSEN; CHERRINGTON, 1983).

Existem evidências do uso dos jogos de empresas como ambiente de pesquisa. Dickinson, Gentry e Burns (2004) realizaram um levantamento que identificou estudos relacionados com o usou dos jogos de empresas para a aquisição de dados para testes empíricos, os primeiros autores que promoveram este tipo de pesquisa foram Cangelosi e Dill (1965) e mais recentemente, foram identificados dois artigos, Mathiew e Schulze (2006) usaram simulação organizacional para testar o desempenho de equipes e outro estudo interessante, caracterizado como um ensaio teórico foi desenvolvido por Hambrick (2007), que defende que os jogos de empresas poderiam ser um importante meio de pesquisa prévia para obtenção de dados e, na sequencia, avançaria em pesquisas em níveis superiores de complexidade.

\section{Estudos de gestão de projetos utilizando jogos de empresas}

A partir de buscas em bases científicas de publicação de artigos, encontrou-se um estudo com alinhamento ao problema deste trabalho. Trata-se de uma publicação no International Journal of Project Management de Andrew Martin no ano de 2000. O artigo descreve pela primeira vez um simulador denominado Contract $\&$ Construct $(\mathrm{C} \& \mathrm{C})$, que foi projetado para ser utilizado em disciplinas de gerenciamento de projetos. O estudo discute a experiência no uso do C\&C e descreve o desenvolvimento de um programa de educação para gestão de projetos de um motor em cursos de MBA (Master in Business Administration) e de Mestrado em Engenharia baseado na simulação dinamizada em um jogo de empresas

O Contract \& Construct foi, inicialmente, desenvolvido para apoiar o ensino de gerenciamento de projetos para um curso de MBA na Warwick Business School, no Reino Unido. No curso, temas como: definição de projetos, de planejamento e de controle de projetos, software de projeto, prevenção de falha e de recuperação, gerenciamento de riscos, precificação do projeto, negociação, controle de custos, organização de projetos, o trabalho e a construção de equipes, gerenciamento de projetos em TI e ambientes de P\&D (Pesquisa e Desenvolvimento) e contratos do projeto. 
O software demonstra a relevância da aplicação prática dos conceitos teóricos fundamentais, proporcionando uma experiência simulada em um ambiente seguro. Por intermédio do seu ambiente interativo e do trabalho em grupo, o C\&C proporciona a formação de equipe e o complemento aos métodos tradicionais de ensino. Ele foi executado tanto como uma contribuição para um curso sobre gerenciamento de projetos, tal como para um recurso autônomo. O objetivo do $\mathrm{C} \& \mathrm{C}$ foi fornecer, de forma dinâmica, uma experiência de gerenciamento de projeto, que envolvesse todas as pessoas. Os destaques do simulador Contract \& Construct, foram:

- o trade-off entre custo, qualidade (ou satisfação do cliente), tempo e segurança para atender determinados objetivos:

- a prática de planejamento do projeto e progressão por meio de tomada de decisão;

- a aplicação de técnicas de gerenciamento de projetos;

- alguns dos elementos do gerenciamento de projetos, tais como gestão de pessoas;

- a necessidade de viver com as consequências de estratégia e de decisões.

Os elementos de gestão clássico de planejamento, de comando, de coordenação e de controle são inerentes à simulação. Outro importante elemento da natureza do jogo é o estilo comportamental de apresentação, que complementa o conteúdo funcional e refletem muitos dos papéis comportamentais gerenciais. Vários jogos de gerenciamento incluem ênfases comportamentais a prática, todavia o $\mathrm{C} \& \mathrm{C}$ não incluir elementos de dramatização. Além disso, os jogadores, na dinâmica de aplicação do jogo, normalmente, são organizados em pequenos grupos, aonde competem com outras equipes em termos de seu desempenho e da cooperação. A competição e a comunicação servem como uma oportunidade para a formação de equipes e para o desenvolvimento de habilidades interpessoais. Sendo o processo de tomada de decisão do grupo um foco de atenção desejado e o papel de formação de equipe o objetivo principal de um cliente. As estruturas de gerenciamento de projetos são representadas em algum nível, e como objetivos finais tiveram a preocupação de dissociar as desvantagens, em projetos, da relação de causa e efeito. Assim, Martin (2000) afirma que o Contract\& Construct constitui uma simulação de gestão significativa.

\section{Método de Pesquisa}

O presente estudo pode ser caracterizado como uma pesquisa descritiva por meio da qual analisa-se a opinião de profissionais de gestão de projetos em uma atividade vivencial na modalidade de um jogo de empresas dinamizado por um simulador desenvolvido sob medida. Esse tipo de pesquisa inclina-se para a descrição de um determinado fenômeno numa situação específica sem maior enquadramento em aspectos subjacentes e detalhamento de um problema.

Quanto aos procedimentos técnicos, o trabalho pode ser caracterizado como um levantamento de opinião que teve como objetivo analisar a impressão de certos indivíduos quanto a um conjunto de questões apresentadas na forma de um instrumento específico, neste caso, um questionário sobre a formação de competências para gestores de projetos.

No intuito de identificar as opiniões dos profissionais houve, com antecedência a elaboração de um questionário com a apreciação da literatura que trata do tema e que sucedeu a operacionalização das variáveis deste estudo.

Da literatura tomaram-se os estudos de Carvalho e Rabechini Jr (2006) que analisaram as habilidades relevantes em gestão de projetos sob três aspectos: o gestor, a equipe e o contexto da organização para o desenvolvimento de projetos. Desse estudo foram adaptadas sentenças para cada uma das dimensões apresentadas pelos autores, deve-se salientar que as questões foram associadas ao contexto do caso desenvolvido para a simulação. No Quadro 1 estão indicadas as dimensões com respectivas variáveis escolhidas para o estudo e questionário aplicado para saber o nível de aprendizado com o simulador. 
Quadro 1: Dimensões e Variáveis de Competências em Projeto Avaliadas

\begin{tabular}{|c|c|}
\hline Din & Variáveis Estudadas e Questionário \\
\hline Indivíduo & $\begin{array}{l}\text { Estabelecer metas e cumpri-las, negociar e gerenciar o negócio do projeto, ações técnicas, estabelecimento } \\
\text { de recursos, gerenciamento do prazo, comunicação e pessoas. } \\
\text { Perguntas do Questionário: } \\
\text { 1) A Simulação com o PMS foi capaz de desenvolver capacidade/habilidade de... } \\
\text { Estabelecer metas e cumpri-las?(v1a); Negociação com várias entidades que participam do projeto?(v1b); } \\
\text { Gerenciamento de projeto?(v1c); Coordenar as ações técnicas do projeto?(v1d); } \\
\text { Estabelecer os recursos necessários às várias fases do projeto?(v1e); Gerenciamento do prazo das } \\
\text { atividades do projeto?(v1f); Identificação e resolução de conflitos no âmbito dos projetos?(v1g); } \\
\text { Estabelecer um sistema de comunicação?(v1h); Gerenciar as relações humanas e estimulação das } \\
\text { pessoas? (v1i) }\end{array}$ \\
\hline Equipe & $\begin{array}{l}\text { Foco no resultado, inovação e criatividade, adaptação, qualidade, desempenho, prazos e orçamentos, } \\
\text { superação de desafios, espírito de equipe. } \\
\text { Perguntas do Questionário: } \\
\text { 2) O nível de desenvolvimento que Simulação com o PMS proporcionou ao ambiente de equipe foi de ... } \\
\text { Orientação para o resultado(v2a); Possibilidades de inovação e desenvolvimento criativo(v2b); } \\
\text { Adaptação às novas situações(v2c); Garantia da qualidade das açôes(v2d); Aprimoramento técnico(v2e); } \\
\text { Consciência dos prazos e orçamentos(v2f); Estímulo favorável às mudanças(v2g); Confiança para } \\
\text { superação do s desafios(v2h); Orientação para o grupo em detrimento ao individualismo(v2i) }\end{array}$ \\
\hline Organização & $\begin{array}{l}\text { Suporte às estratégias de projetos, apoio político, avaliações periódicas, fornecimento de recursos, } \\
\text { disponibilidade de informação, monitoramento, divulgação dos resultados, institucionalização a } \\
\text { aprendizagem em projetos. } \\
\text { Perguntas do Questionário: } \\
\text { 3) Projetando a experiência da equipe simulada no PMS para uma realidade, pode-se considerar que... } \\
\text { As estratégias e planos do projeto foram estabelecidos.(v3a); O desempenho poderia ser mais eficiente } \\
\text { se fosse inclúda uma variável de apoio político ao projeto no nível executivo(v3b); O projeto foi } \\
\text { adequadamente iniciado(v3c); Houve estimulo para avaliações periódicas do projeto durante suas fases. } \\
\text { (v3d); Houve garantia que os recursos foram fornecidos.(v3e); Faz-se necessário o apoio especializado } \\
\text { em determinadas situações(v3f); Exercitou a transição do projeto para a operação (v3g); Houve a } \\
\text { divulgação dos resultados do projeto(v3h); Existiu o compartilhamento da aprendizagem obtida no } \\
\text { projeto entre as equipes(v3i) }\end{array}$ \\
\hline
\end{tabular}

\section{Fonte: Elaborado pelo Autor, 2014}

Para a coleta de dados criou-se um questionário estruturado dividido em duas partes. A primeira com informações sócio-profissionais como idade, formação e tempo de experiência na área de projetos e a segunda com as questões sobre a formação de competências em gestão de projetos.

$\mathrm{Na}$ segunda parte do questionário as questões foram divididas entre as três dimensões e cada uma delas com nove sentenças, totalizando 27 questões. A escala destas questões aproximou-se da escala forçada de classificação com 10 pontos que segundo Malhotra (2001) são escalas que forçam os pesquisados a manifestar uma opinião sem a opção de respostas de não conhecimento. Tal escala foi escolhida para sugerir aos respondentes, que são profissionais da área, o pensar de forma objetiva em uma escala de zero a dez.

Aplicou-se o questionário, primeiramente, em treinamento na própria empresa, tratou-se essa capacitação como piloto e não houve dados analisados, todavia, serviu como aplicação das propostas numa área específica e como teste para avaliar a viabilidade da ACP (Abordagem Centrada no Participante ou Student Centred-Learning) e do simulador organizacional desenvolvido. Com a análise da consistência interna deste instrumento, ajustou-se o questionário para a ACP posterior..

A Abordagem Centrada no Participante permite que os alunos envolverem-se ativamente nos processos de aprendizagem. Os partícipes passam todo o tempo de aula buscando a construção de uma nova compreensão do material de forma pró-ativa. Uma variedade de atividades práticas são administradas, a fim de promover 
a aprendizagem bem-sucedida. Isto é, estilos de aprendizagem distintos são incentivados no treinamento, para isso, deve-se proporcionar ferramentas variadas para os alunos, tais como: metodologias a tarefas e aprendizagem consciente. Cria-se, assim, um melhor ambiente para aprendizado SMITH (2003).

Segundo Malhotra (2001) a confiabilidade de consistência interna trata-se de uma abordagem para avaliar a competência de um conjunto de itens, quando vários são somados para formar uma medida total para a escala. O teste utilizado foi o alfa de Cronbach que trata da média de todos os coeficientes que resultam das diferentes maneiras de dividir ao meio os itens da escala. Este coeficiente varia de zero a um e o valor de 0,6 ou menos indica uma confiabilidade de consistência interna insatisfatória.

O resultado do alfa de Cronbach para as respostas do questionário aplicado nesse estudo foi de 0,98 demonstrando a confiabilidade da consistência interna dos padrões de respostas, sugerindo, desta maneira, que os pesquisados compreenderam o que lhes havia sido perguntado.

No que se refere ao tratamento dos dados, o estudo usou uma abordagem quantitativa que segundo Malhotra (2001) utiliza a quantificação de dados e a aplicação de alguma análise estatística.

Adotou-se a mediana como estatística para ilustrar as diferenças de opinião entre os grupos de profissionais pesquisados, assim foi possível, por meio de uma análise gráfica, observar o comportamento das respostas quanto à contribuição do ACP para a formação de competência em projetos. Para realização de inferências, utilizou-se de análise univariada com o teste t de student com o intuito de comparar as médias das respostas dos grupos de pesquisados.

\section{Descrição da Pesquisa}

Esta seção destina-se no detalhamento das etapas que compuseram a pesquisa, apresentado não somente a organização dos procedimentos metodológicos do estudo, mas, também, descrevendo o contexto no qual o estudo estava inserido.

A motivação para a pesquisa emergiu durante a proposta de elaboração de uma capacitação para a equipe de projetos de um setor de apoio da indústria automobilística instalada no estado do Rio de Janeiro. $\mathrm{Na}$ ocasião, o interesse da empresa era um treinamento voltado para a assimilação do modelo de gestão de projetos que a organização desenvolveu ao longo dos anos, contudo, por diversas razões, a aplicação na unidade brasileira estava tendo dificuldades na incorporação dos conceitos.

Os solicitantes estavam inclinados em apoiar uma capacitação de caráter prático e que pudesse estimular os participantes a refletir sobre os fundamentos da gestão de projetos. Deste modo, surgiu a proposta da ACP com um simulador customizado envolvendo situações da gestão de projetos da empresa. Várias reuniões foram necessárias para criar o caso simulado, para determinar as variáveis do simulador, para elaborar os indicadores de resultados próximos aos utilizados e para planejar do treinamento.

Nesta fase, que se denominou de ACP Piloto, foi desenvolvido o simulador PMS-SIM, a atividade vivencial (ACP) e o instrumento de opinião dos participantes quanto à contribuição da vivência para a formação de competências em gestão de projetos. Ao final do treinamento a reação dos participantes foi tão positiva que os responsáveis da empresa solicitaram uma nova edição. A boa repercussão motivou os instrutores a realizarem melhorias no simulador e nos demais recursos que compunham a ACP e desta experiência surgiu à ideia de realizar uma pesquisa estruturada.

Após a fase de ACP Piloto a pesquisa foi organizada para coletar as impressões de um grupo de profissionais da área de projetos da empresa com as mais diversas características em termos de formação, de cargo e de experiência. A Figura 1 ilustra as etapas da pesquisa. 
Figura 1: Etapas da Pesquisa

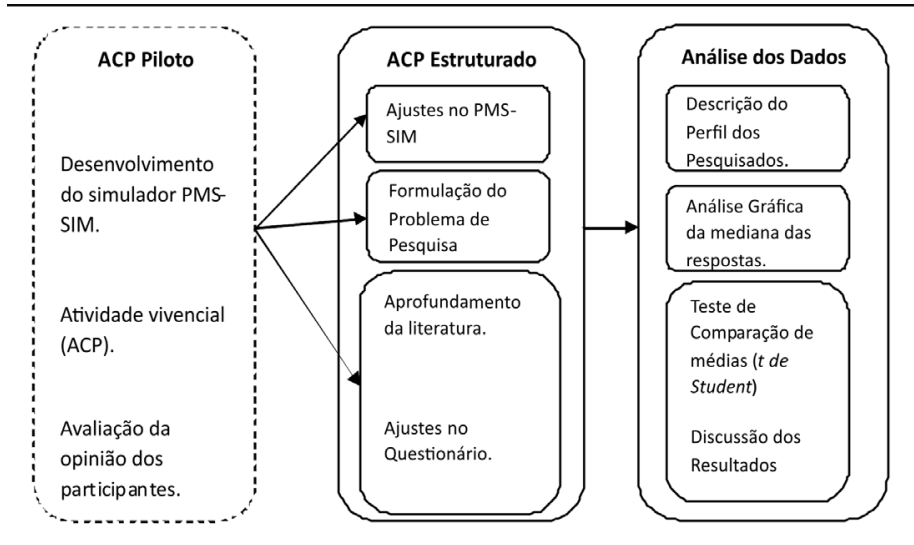

Fonte: Elaborado pelo autor

A atividade vivencial estruturada, além dos ajustes técnicos e melhorias no simulador, foram fundamentadas num problema formal de pesquisa que guiou todo o estudo, para tanto a literatura específica do tema foi aprofundada, instrumento de coleta de dados recebeu ajustes de acordo com as referências pesquisadas, houve um plano de aplicação da atividade e durante a mesma os dados junto aos profissionais foram coletados.

Com os dados coletados o perfil dos participantes foi avaliado, as respostas foram tratadas tanto de forma descritiva com comportamento da mediana como também foram realizados testes de comparação de médias para verificar a diferença entre os grupos de profissionais de projetos pesquisados, com isso foi possível realizar a análise e discussão dos resultados.

\section{Descrição do simulador organizacional}

Para aplicação da atividade Oliveira e Teodoro (2013), desenvolveram um simulador organizacional denominado PMS-SIM (Project Management Simulation). Esse simulador tem o objetivo de oportunizar que profissionais de equipes de projetos possam aplicar as competências estudadas em treinamentos de gerenciamento de projetos, de modo integrado, em um ambiente lúdico, de modo que proporcione a vivência do trabalho em equipe. A simulação com o PMS-SIM reforça e estimula:

- As habilidades técnicas em gestão de projetos (Escopo, Prazo, Custo, Recursos
Humanos, Qualidade, Comunicação, Risco e Integração);

- As habilidades gerenciais, como liderança, negociação, comunicação;

- O trabalho de equipe.

A simulação do PMS-SIM objetiva proporcionar a vivência sobre o ciclo de vida de gerenciamento de um projeto (iniciação, planejamento, execução/ controle e encerramento), criando no participante uma consciência da importância do bom gerenciamento em qualquer projeto. Os partícipes vivenciam a elaboração do Plano do Projeto, desenvolvendo, a partir do Project Charter, as seguintes atividades: Estrutura Analítica de Projetos (EAP), Cronogramas, Orçamento, Análise dos Indicadores de Qualidade, Plano de Encerramento do Projeto e Matriz de Maturidade em Projetos.

Os aspectos básicos da simulação tratam de um projeto de automóvel com dois modelos. Os elementos centrais do caso simulado são: escopo, prazo, custo e qualidade.

A atividade de simulação compreende as cinco etapas de um projeto, sendo duas de apresentações e três de práticas gerenciais simuladas: (a) iniciação com a apresentação do caso simulado, (b) planejamento do projeto, (c) execução e (d) controle (nas fases de execução e de controle do projeto, os participantes simulam a execução do principal produto, emitindo relatórios de desempenho e fazendo os ajustes necessários) e (e) na finalização realiza-se o encerramento do projeto e analsa-se os principais indicadores de desempenho. Nessa fase acontece o enceramento do Projeto,

Em cada rodada há resultados das fases por meio de indicadores das atividades de escopo, de prazos, de custos e de qualidade.

\section{Perfil dos pesquisados}

Dos 25 participantes do ACP (Abordagem Centrada no Participante) 21 responderam o questionário proposto no estudo. A descrição do perfil dos pesquisados pode ser observado na Tabela 1: 
Tabela 1: Perfil dos pesquisados

\begin{tabular}{lccccccc}
\hline & Qtd & Média & Média & \multicolumn{4}{c}{ Formação } \\
\cline { 5 - 7 } Idade & Experiência & Administração & Engenharia & Contabilidade & Economia \\
Gerentes & 3 & 45 & 10 & - & $100 \%$ & - & - \\
Coordenadores & 6 & 35 & 6 & $16 \%$ & $84 \%$ & - & - \\
Especialistas & 7 & 33 & 6 & - & $72 \%$ & $14 \%$ & $14 \%$ \\
Analista & 5 & 31 & 3,5 & $20 \%$ & $80 \%$ & - & - \\
\hline
\end{tabular}

Fonte: Elaborado pelo autor, 2013.

Percebe-se que a quantidade de cada grupo hierárquico é equilibrada, sendo três gerentes, seis coordenadores, sete especialistas e cinco analistas. A maior média de idade pertence ao cargo mais elevado (gerente) com 45 anos e a menor média pertence aos analistas, menor nível hierárquico na equipe de projetos.

Com relação à experiência na área de projetos, novamente, prevalecem os gerentes com 10 anos de média, coordenadores e especialistas com 6 anos e analistas com 3,5 anos de experiência. Finalizando o perfil dos pesquisados, verifica-se que, em sua maioria, o grupo era formado por engenheiros, todavia, pode-se destacar a presença de profissionais com formação em administração, em ciências contábeis e em economia.

\section{Análise dos resultados}

\section{Análise geral das competências proporcionadas na ACP com o PMS-SIM}

Os cargos são uma "proxi” (critério pré-estabelecido) para a distribuição dos pesquisados em grupos específicos. Adotou-se, para análise das médias das opiniões dos participantes o teste t de student que objetiva a comparação das médias de amostras. Esse teste demonstrou que as médias das opiniões dos participantes foram diferentes. A Tabela 2 apresenta os resultados unificados dos testes para cada um dos grupos.

Tabela 2: Teste t da opinião dos grupos de participantes sobre as variáveis de competências estimuladas pela ACP com o PMS

\begin{tabular}{|c|c|c|c|c|c|}
\hline & Xgc* & GERENTES & COORDENADORES & ESPECIALISTAS & ANALISTAS \\
\hline Gerentes & 6,85 & - & & & \\
\hline Valor-P & & - & & & \\
\hline Coordenadores & 4,68 & $\begin{array}{c}\text { Médias } \\
\text { Diferentes }\end{array}$ & - & & \\
\hline Valor-P & & $5,06 \mathrm{E}-11$ & - & & \\
\hline Especialistas & 5,11 & $\begin{array}{c}\text { Médias } \\
\text { Diferentes }\end{array}$ & Médias Diferentes & - & \\
\hline Valor-P & & $1,31 \mathrm{E}-15$ & 0,0365 & - & \\
\hline Analistas & 5,63 & $\begin{array}{c}\text { Médias } \\
\text { Diferentes }\end{array}$ & Médias Diferentes & Médias Diferentes & - \\
\hline Valor-P & & $6,35 \mathrm{E}-10$ & 0,0002 & 0,00014 & - \\
\hline
\end{tabular}

Nível de Significância 99\% Unicaudal. ${ }^{*}$ Xgc $=$ Média Geral das Variáveis de Competências

Fonte: Elaborado pelo autor, 2013. 
Verifica-se na Tabela 2, as médias de todas as respostas por cargo. De posse desse resultado, confirmouse a a "proxi”" estabelecida, ou seja, existe diferença entre as opiniões de cada cargo. Utilizando o teste-t e relacionando todas as possibilidades de igualdade entre os grupos, pode-se sugerir que os grupos apresentam características e opiniões distintas por apresentarem fatores como nível de experiência, cargo e idades diferentes.

\section{Análise das Dimensões de Competências}

Sabendo-se que existe diferença entre as médias gerais das opiniões dos participantes acerca das variáveis de competências estimuladas pela vivencia, analisouse as dimensões do questionário adotado nesse estudo, dividindo-o em formações individuais (D1), de equipes (D2) e de organização (D3), seguindo a divisão proposta por Frame (1999) e por Carvalho e Rabechini Jr. (2006; 2015). Para tanto, analisouse a média geral dos grupos e utilizou-se o radar da mediana das questões de cada dimensão. Ressaltase que o radar da mediana é composto das questões apresentadas, anteriormente, no Quadro 1 do tópico método de pesquisa e explicitou-se cada questão, no radar, por meio dos códigos.

Figura 2: Gráfico radar das respostas das questões da dimensão individual (D1)

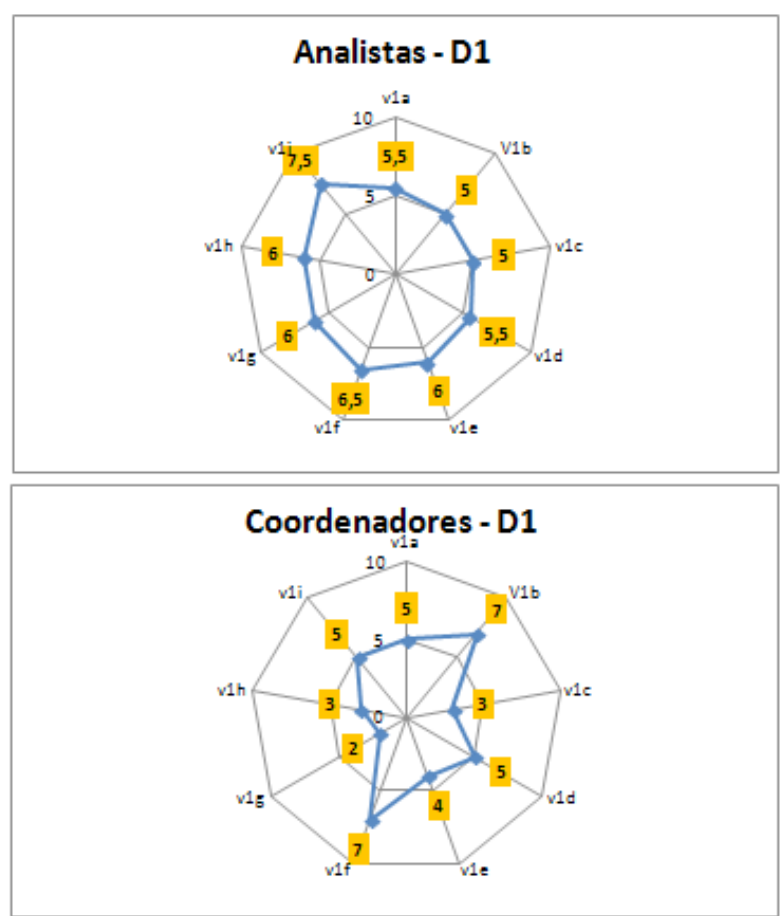

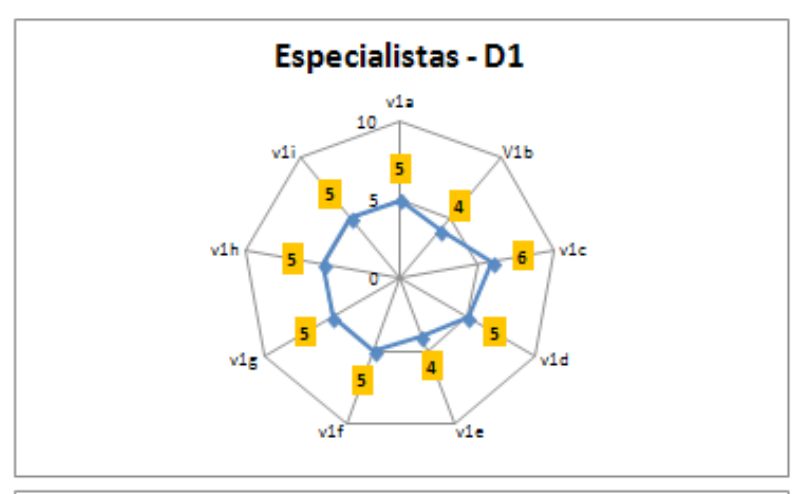

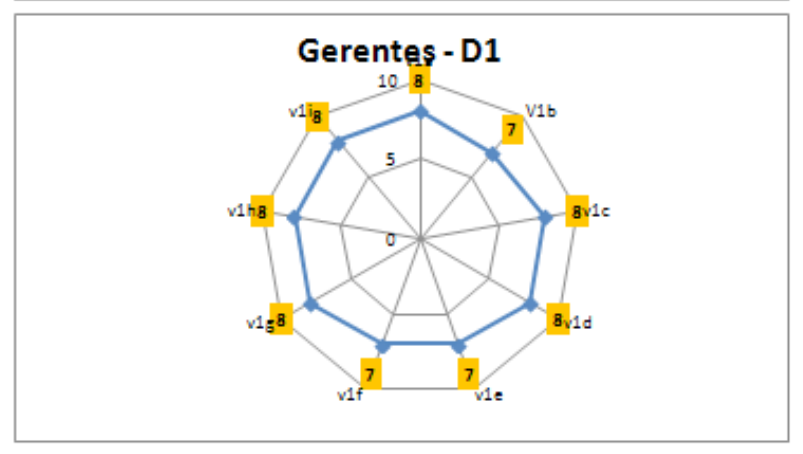

Fonte: Elaborado pelo autor, 2013.

\section{Dimensão Individual (D1)}

$\mathrm{Na}$ dimensão individual verifica-se que as respostas dos gerentes (média 6,85) são estatisticamente diferentes, a um nível de significância de 0,01 , das opiniões dos demais profissionais. As respostas dos coordenadores (média 4,44) podem ser consideradas iguais as dos especialistas (média 5,17) e dos analistas (média $5,74)$. Contudo, as opiniões entre os especialistas e os analistas foram diferentes.

Para analisar, de forma minuciosa, a dimensão individual, criou-se um gráfico radar para cada cargo, usando a mediana das respostas de cada questão, conforme a Figura 2.

Utilizou-se a mediana, estatística que representa uma medida associada à posição que não sofre $\mathrm{o}$ efeito acentuado da tendência central como o caso da média, para melhorar a discriminação dos dados. Sabe-se que ela representa o ponto intermediário de uma distribuição de dados, que no caso estudado, representam as respostas dadas pelos participantes a cada pergunta.

Analisando os resultados identificou-se que os analistas avaliam positivamente a interação do jogo com o gerenciamento das relações humanas e com a estimulação das pessoas (7,5 - v1i). Já os 
especialistas acreditam que a interação do jogo para desenvolvimento de competências individuais é regular. Para os coordenadores as competências destacadas foram à gestão do prazo $(7$ - v1f $)$ e a negociação entre entidades do projeto $(7-\mathrm{v} 1 \mathrm{~b})$ e os gerentes concluem que o jogo tem capacidade de desenvolver competências individuais em todos os pontos pesquisados.

Nas dimensões seguintes são utilizadas as mesmas descrições adotadas para análise das opiniões e de suas variáveis de composição.

\section{Dimensão Equipe (D2)}

$\mathrm{Na}$ dimensão de formação de competências para equipe, as respostas dos gerentes (média 6,81), também, apresentaram diferença estatística das opiniões dos outros profissionais. No entanto, as respostas dos coordenadores (média 4,84), dos especialistas (média 5,13 ) e dos analistas (média 5,65), para essa dimensão, podem ser consideradas iguais.

Os resultados indicam que os coordenadores, os especialistas e os analistas tendem a responder as questões de forma similar. Os especialistas analisaram a dinâmica do jogo para o desenvolvimento de competências, de forma regular, somente a questão relacionada a possibilidades de inovação (4 - v2b) obteve avaliação inferior. Porém, os analistas destacam a orientação do jogo para o trabalho em equipe (v2i), tendo essa questão mediana sete.

Os coordenadores destacaram o estímulo as mudanças (7 - v2g), como um ponto de importante da ACP. Os gerentes, mais uma vez, tiveram respostas com mediana superior aos demais cargos, tendo um único ponto com avaliação seis, relacionado a orientação que a equipe deu aos resultados (v2a).

\section{Dimensão Organizacional (D3)}

Para a dimensão contribuição, mais uma vez, as respostas dos gerentes (média 6,89) foram diferentes aos demais cargos. Entretanto, considerou-se, estatisticamente, as respostas dos coordenadores (média 4,73), dos especialistas (média 5,11) e dos analistas (média 5,50) iguais.

Com os resultados das respostas, observou-se que os coordenadores, os especialistas e os analistas tendem a responder as questões de forma similar e com pouca diferença entre si. Os especialistas destacaram a divulgação dos resultados do projeto (8 - v3h), por parte da organização, sendo as outras questões pontuadas de forma regular. Já os analistas destacaram o compartilhamento de aprendizagem pelas equipes $(7-v 3 i)$.

Os coordenadores não destacaram nenhuma questão acima do padrão de respostas dos demais cargos, sendo as respostas das questões sempre regulares. Não obstante, os gerentes avaliaram as questões sobre a dimensão organizacional sempre acima do padrão, destacando três variáveis com mediana de valor oito, são: as garantias de fornecimento dos recursos (v3e), o apoio especializado em determinadas situações (v3f) e a divulgação dos resultados (v3h).

\section{Conclusão}

As opiniões dos profissionais de projetos revelaram a existência de distintas impressões quanto às contribuições da ACP na formação de competências. Percebe-se que as características dos grupos de profissionais de acordo com o perfil associado, a experiência e o cargo, determinam uma avaliação diferente.

De forma geral, os participantes indicaram que a ACP dinamizada pelo jogo de empresas promove contribuições na formação de competências em projetos, apesar das distintas opiniões, sendo sempre sua mediana, maior parte das respostas, superior a 5 em uma escala de 0 a 10.

Os gerentes de projetos avaliaram a contribuição da ACP em níveis superiores aos demais, revelando que os mais experientes e de maior nível hierárquico percebem uma consistente contribuição do jogo de empresas na formação de competências. Esses profissionais, por já possuírem uma visão integrada da gestão de projetos, avaliaram a atividade proposta como uma exercício que simula a gestão em projetos.

De maneira similar, os coordenadores, os especialistas e os analistas indicaram que a ACP promoveu contribuições moderadas nas três dimensões analisadas. Cabendo destaque para contribuições acima da média, como: o gerenciamento das relações humanas na dimensão indivíduo (D1), o estimulo 
a mudanças e o estabelecimento de estratégias e de planos para os projetos na dimensão equipe (D2) na dimensão organização (D3) o destaque foi a capacidade de divulgação dos resultados do projeto.

Com os resultados obtidos verificou-se que o objetivo do estudo foi alcançado e a análise proporcionou um panorama descritivo com as distintas opiniões dos participantes, nada obstante, de maneira geral, a ACP proporcionou contribuições de moderadas a superiores na formação de competências.

O estudo propicia contribuições interessantes nos mais diversos aspectos para o campo da gestão de projetos e da aprendizagem gerencial que pode ser assim listadas:

- A descrição de uma atividade vivencial com profissionais da área de projetos e com o desenvolvimento de um simulador organizacional específico para a capacitação;

- Realização de uma aplicação prática de gestão de projetos e alinhando ao modelo de levantamento de competências exigidas para a carreira em projetos;

- Demonstroação do potencial dos jogos de empresas para a formação na área de gestão de projetos, contextualizado para a realidade de uma empresa automobilística;

- Fundamentação teórica com a compilação das principais abordagens em competências para gestores de projetos. merece atenção a natureza da escala de resposta do questionário na produção de dados para análise quantitativa, pois as notas num intervalo de zero a dez, se consideradas escalas ordinais, não poderiam ser tratadas com uso de testes paramétricos.

Das limitações, surgem proposições para avanços na pesquisa e para novos estudos. Sugerem-sd aos interessados no tema uma agenda de pesquisa, com destaque:

- Comparar o desempenho das equipes no jogo de empresas com as respostas ao questionário sobre a formação de competências na gestão de projetos;

- Promover uma pesquisa quanto à eficiência educacional do ACP realizado, medindo a validade interna da atividade, ou seja, se os participantes melhoram sua aprendizagem após o jogo de empresas. Isto poderia ser feito com aplicação de testes antes e depois da atividade vivencial;

- Realizar uma pesquisa qualitativa por meio de entrevistas ou grupos focais com os participantes;

- Verificar a natureza dos dados quanto sua normalidade e homogeneidade de variância, no caso da não adequação, sugerem-se o uso de testes não paramétricos para análise, como o teste de Mann-Whitney.
No âmbito empresarial possibilitou-se ilustrar o uso de simuladores organizacionais em treinamentos para gestores de projetos, trazendo algo além da exposição do conhecimento (método convencional), promovendo a aplicação de suas competências num ambiente simulado.

O estudo apresenta limitações que merecem reflexões, como: a não utilização dos resultados do jogo de empresas na comparação com as opiniões dos participantes, o uso questionável da opinião de indivíduos que poderiam estar enviesados ou até mesmo o fornecimento de informações que não refletem a sua real percepção da experiência. Ainda

\section{Referências}

BARTSCH, V.; EBERS, M.; MAURER, I. Learning in project-based organizations: The role of project teams social capital for overcoming barriers to learning. International Journal of Project Management, v.31, p.239-251, 2013.

BIBLIA SAGRADA, Nova Versão Internacional. São Paulo: Editora Vida, 2003.

BUGANZA, T.; KALCHSCHMIDT, M.; BARTEZZAGHI, E.; AMABILE, D. Measuring the impact of a major project management educational program: The PMP case in Finmeccanica. 
International Journal of Project Management, n.31, p. 285-298, 2013.

CANGELOSI, V.E; DILL, W.R. Organizational Learning: Observation Toward a Theory. Administrative Science Quarterly, v. 10, n.2, p. 175-203, 1965.

CARVALHO, M. M.; RABECHINI JR, R. Concepção de um programa de gerência de projetos em instituição de pesquisa. Revista Valenciana d'Estudis Autonòmics, v.1, n.20, p. 1, 1999.

CARVALHO, M. M. Construindo competências para gerenciar projetos: teoria e casos. São Paulo: Atlas, 2006.

CARVALHO, M. M.; RABECHINI JR, R. Perfil das competências em equipes de projetos. RAEeletrônica, São Paulo, v.2, n.1, p. 1-17, 2003.

CARVALHO, M. M. Fundamentos em Gestão de Projetos: Construindo Competências para gerenciar projetos. São Paulo: Atlas, 2015.

DICKINSON, J.R., GENTRY, J.W; BURNS, A.C. A Seminal Inventory of Basic Research Using Business Simulation Games. Development in Business Simulation and Experiential Learning, v. 31, p. 345351, 2004. Anais. CD-ROM.

FLEURY, A. C. C.; FLEURY, M. T. L. Construindo o Conceito de Competência. Revista de Administração Contemporânea (RAC), Edição Especial, 2001.

FLEURY, A. C. C.; FLEURY, M. T. L. Estratégias empresariais e formação de competências. São Paulo: Atlas, 2004.

FRAME, J.D. Project management competence: building key skills for individuals, team and organizations. San Francisco: Jossey-Bass, 1999.

FREEMAN, J.M., DUMAS P. Business games: from business schools to business firms. In: Crookall D., Saunders D, editors. . Communication and simulation: from two fields to one theme. Clevedon, England: Multilingual Matters Ltd. p. 201-213, 1989.

GILBREATH, R.D. Working with pulses, not streams: using projects to capture opportunity. In: Cleland D, King W, editors. Project management Handbook. NewYork: Van Nostrand Reinhold, p.3, 1988.

HAMBRICK, D.C. Upper Echelons Theory: An
Update. Academy of Management Review, v. 32, n. 2, p. 334-343, 2007.

HOLT, D.H. Management: principles and practices. Englewood Cliffs, NJ: Prentice Hall International, 1987.

JENSEN, R.; CHERRINGTON, D. J. The Business Management Laboratory. New York. McGraw-Hill Higher Education, 1983.

KEYS, B.; WOLFE, J. The role of management games and simulations in education and

research. Journal of Management, v. 16, n. 2, p. 307-336, 1990.

KOTTER, J. P. What effective managers really do. Harvard Business Review, v. 60, p.156-167, 1982.

WIRTH, I. Project management education: current issue sand future trends. International Journal of Project Management, v.10, n1, p. 49-54, 1992.

LARRECHE, J.C. On simulations in business education and research. Journal of Business Research, v.15, n. 6, p. 559-571, 1987.

MALHOTRA, N. Pesquisa de Marketing; uma orientação paliçada. $3^{\mathrm{a}}$ Ed. Porto Alegre, Bookman, 2001.

MARTIN, A. A simulation engine for custom project management education. International Journal of Project Management, v. 18, n. 3, p.201-213, 2000.

MATHIEU, J.L; SCHULZE, W. The Influence of Team Knowledge and Formal Plans on Episodic Team Process-Performance Relations. Academy of Management Journal, v. 49, n.3, p. 605-619, 2006.

McCLELLAND, D. C.. Testing for Competence Rather Than for "Intelligence". American Psychologist. Havard University, January, 1973.

MOTTA, G. S.; QUINTELLA, R. H. ; MELO, D. R. A. Jogos de empresas como componente curricular: análise de sua aplicação por meio de planos de ensino. Revista Organização \& Sociedade, Salvador, v. 19, n. 62, 2012.

NOVEMBER P. Right and left brain marketing education. Journal of Marketing Education, Summer, v.15, n. 2, p. 3-12, 1993.

OLIVEIRA, M. A. Implantando o laboratório de gestão: um programa integrado de educação gerencial 
e pesquisa em administração. 2009. Tese (Doutorado em Administração) - Faculdade de Economia, Administração e Contabilidade, Universidade de São Paulo, São Paulo, 2009a. Disponível em: <http:// www.teses.usp.br/teses/disponiveis/12/12139/ tde-18122009-094527/>.

OLIVEIRA, M. A. Heurísticas e Vieses de Decisão: Um Estudo com Participantes de uma Simulação Gerencial. Sociedade, Contabilidade e Gestão (UFRJ), v. 4, n. 1, p. 72-90, 2009b.

OLIVEIRA, M. A.; TEODORO, P. Manual do Simulador de Gestão de Projetos (PMS-SIM). Rio de Janeiro, RJ, Fundação Biblioteca Nacional. N. de Registro 602.412, Maio, 2013.

PETERS L.A.; HOMER J. Learning to lead, to create quality, to in fluence change in projects. Project Management Journal, v. 27, n. 1, p. 5-11, 1996.

PROJECT MANAGEMENT INSTITUTE (PMI). A guide to the project management body of knowlegde (PMBoK). 4. ed. Project Management Institute, 2008.

PROJECT MANAGEMENT INSTITUTE (PMI). A guide to the project management body of knowlegde (PMBoK). 5. ed. Project Management Institute, 2013a.

PROJECT MANAGEMENT INSTITUTE (PMI). About Us. Disponível em <http://www.pmi.org > Acesso em 30 Nov. de 2013b.

PROJECT MANAGEMENT INSTITUTE (PMI). PMI's 2012 Annual Report Enhancing Business Value Around the World. Disponível em: <http:// www.pmi.org>. Acesso em 30 Nov. de 2013c.

SAUAIA, A. C. A. Laboratório de gestão: simulador organizacional, jogos de empresas e pesquisa aplicada. Barueri, SP: Manole, 2008.

SAUAIA, A. C. A ; OLIVEIRA, M. A. Decomposição do Desempenho Organizacional em um Jogo de Empresas. Revista Eletrônica de Estratégia \& Negócios, v. 4, n. 1, p.158-182, 2011.

SAUAIA, A. C. A.; ZERRENNER, S. A. Jogos de empresas e economia experimental: um estudo da racionalidade organizacional na tomada de decisão. Revista de Administração Contemporânea, Curitiba, v.13, n.2, 189-209, 2009.
SMITH, M. K. Learning theory: models, product and process. Disponível em: http://infed.org/ mobi/learning-theory-models-product-and-process. Acesso em 01 set. 2014.

WATERIDGE, J. Training for is/it project managers: away forward. International Journal of Project Management. v. 15, n. 5, p. 283-288, 1997. 